\title{
Multiple-relaxation-time lattice Boltzmann simulation of natural convection flow in a partitioned cavity using GPU computing
}

Cite as: AIP Conference Proceedings 2121, 030017 (2019); https://doi.org/10.1063/1.5115862

Published Online: 18 July 2019

Md Jahidul Haque, Md. Mamun Molla, Nasrin Akhter, and Suvash C. Saha

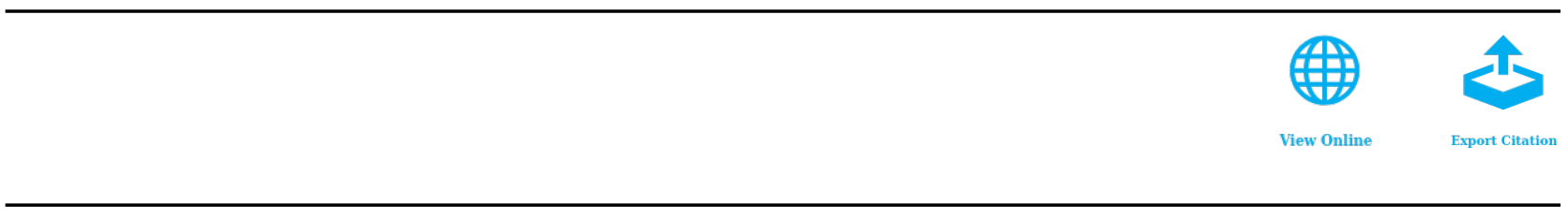

\section{Conference Proceedings}

Get $30 \%$ off all print proceedings!

\section{Enter Promotion Code PDF30 at checkout}

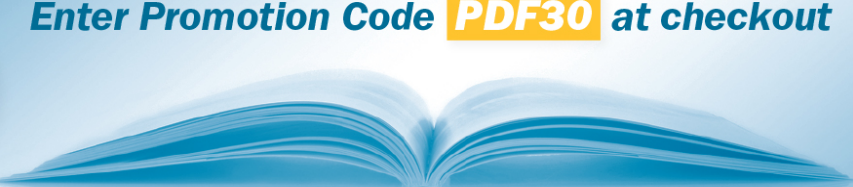




\title{
Multiple-relaxation-time lattice Boltzmann simulation of natural convection flow in a partitioned cavity using GPU computing
}

\author{
Md Jahidul Haque ${ }^{1, b)}$, Md. Mamun Molla ${ }^{2, a)}$, Nasrin Akhter ${ }^{3}$ and Suvash C Saha ${ }^{4}$ \\ ${ }^{1}$ Department of Mathematics Physics, North South University, Dhaka-1229, Bangladesh \\ ${ }^{2}$ Department of Mathematics Physics, North South University, Dhaka-1229, Bangladesh \\ ${ }^{3}$ Department of Mathematics, Dhaka University of Engineering and Technology, Dhaka, Bangladesh \\ ${ }^{4}$ School of Mechanical and Mechatronic Engineering, University of Technology Sydney, Sydney, Australia \\ a)Corresponding author: mamun.molla@northsouth.edu \\ b)jahidul.haque@northsouth.edu
}

\begin{abstract}
In this paper, we demonstrated the implementation of General Purpose Graphics Processing Unit (GPGPU) programming in Compute Unified Device Architecture (CUDA) C for the simulation of natural convection flow in a side-heated three-dimensional (3D) rectangular cavity with a partition. In the present lattice Boltzmann method (LBM) D3Q19 multiplerelaxation-time (MRT) and D3Q6 single relaxation-time (SRT) model are implemented for the simulation of fluid flow and temperature phenomena, respectively. The parallel code is validated with the benchmark problem of a side heated cubic cavity. The results are presented by the temperature distribution in terms of isotherms, local and average Nusselt number and $3 \mathrm{D}$ view of iso-surface for the different Rayleigh number $(R a)$ and the Prandtl number fixed at $\operatorname{Pr}=0.71$. It is also observed that the present parallel implementation of the MRT-lattice Boltzmann simulation in GPU has a substantial computational efficiency rather than the sequential programming in central processing units (CPU).
\end{abstract}

\section{INTRODUCTION}

Innovations in experimental and numerical studies for simulating thermal flows have been improved in a notable attraction over the past few decades. Due to the introduction of various modern sensing technologies as well as the development of new numerical methods and programming tools, different 3D convective flow phenomena are now being simulated at larger grid allocation. Natural convection in a 3D geometry involves the laminar and turbulent flow patterns due to the heat transfer between the heated and cold portion of the indoor environment. Detecting the detail representation of turbulent flow patterns can be crucial in designing ducts and natural air conditioning systems for the thermal comfort inside indoor places. Natural convection in an indoor environment is a common phenomenon to study the thermal comfort inside the building in urban areas. Convective flow analysis in industrial applications can be very incremental for developing better heat distributions in various types of open and closed spaces. The natural convection is done by equilibrating the temperature difference between the heated and cold part inside a cavity. The common phenomena of heat transfer between the heated and cold source can be simulated using the experimental sensing tools or the computer-aided simulations. Tian and Karayiannis [1] conducted an experimental study on a square cavity filled with air by using proportional-integral-differential (PID) for controlling the temperature inside the cavity and low noise K-type thermocouples. Leong et al. [2] studied natural convection for a side heated cubic cavity for different inclination angles and calculated experimental Nusslet numbers for the cavity. Despite the accuracy of the experimental results of natural convection on different simple and complex geometries the numerical simulation of convective flows become more popular due to the low setup cost and minimal time to simulate the phenomena. Moreover, numerical simulations of convective flow using high-performance computers often agreed with the experimental solutions at very good accuracy. Among all the various computational fluid dynamics (CFD) methods, Navier-stokes (NS) based simulations have been the most used method for solving fluid flow problems for a

8th BSME International Conference on Thermal Engineering

AIP Conf. Proc. 2121, 030017-1-030017-7; https://doi.org/10.1063/1.5115862

Published by AIP Publishing. 978-0-7354-1861-5/\$30.00 
wide range of engineering applications. However, the lattice Boltzmann method became renowned as a more sizable numerical method than the formal N-S based methods in recent times.

LBM solves the fluid flow in terms of distribution of fluid particles inspired from the concepts of lattice gas cellular automata (LGCA) by Frisch et. al [3] which was initially developed for solving fluid flows using the Boltzmann's gas kinetic theory. Later different researchers $[4,5]$ developed more refined versions of LBM for simulating 2D and 3D incompressible flow phenomena for various engineering domain. Guo et al. [6] proposed a couple of LBGK model incorporating the BGK collision operator from the BGK model of [7] and the model is capable of solving convective heat phenomena for $2 \mathrm{D}$ or the $3 \mathrm{D}$ cases. Moreover, the heat transfer in natural convection solved as a passive scalar advected by the fluid while the fluid continuity is solved in the velocity space using different lattice models of LBM. The common lattice models for solving temperature are $D 3 Q 6, D 3 Q 15$ and $D 3 Q 19$ while most of the SRT based LBM methods use $D 3 Q 6$ lattice configuration for solving the convective flow phenomena by LBM. Moreover, the evolution equation for achieving the macroscopic energy equation by LBM coupled model of Guo et al. [6] is a popular numerical solution of solving convective flow for various simple and complex geometries. In this paper, we used D3Q6 lattice model for solving the temperature of the fluid regime using the coupled LBGK method.

The most popular LBM methods are based on a single relaxation time(SRT) concepts where fluid continuity is solved through distribution functions using a single relaxation parameter in the velocity space. Furthermore, this solution suffers unstable flow simulations at high Rayleigh number $(\mathrm{Ra})$ and Reynolds numbers(Ra). To mitigate the problems in SRT based LBM solutions, d'Humieres et al. [8] presented a multiple-relaxation-time(MRT) model of LBM for 3D incompressible flow and achieved very stable flow in high Reynolds numbers. MRT based LBM model is very suitable for developing laminar and turbulent flow using high-performance hardware with parallel computation abilities. Very recently, Molla et al.[9] have implemented the GPU based 2D MRT LBM and applied for the lid-driven cavity flow with high Reynolds number as well as the natural and mixed convection flow for the porous media with the high Rayleigh numbers. They found that MRT LBM is very much efficient for the larger grid size problems. However, MRT based model have higher computational cost than the SRT based model which makes the model slower in simulating fluid flows in cases of running the simulation programs in conventional CPU based sequential computing hardware.

Furthermore, GPU based LBM programs are nowadays becoming popular for simulating fluid flows. In recent years, the lattice Boltzmann method (LBM) become popular as a promising alternative of the NS solvers due to the flexibility and the simplicity of the technique. LBM is also capable of parallel implementation of the grid which can incredibly increase the simulation process. Previously, high-performance computers(HPC) perform sequential programming of LBM algorithms to simulate fluid flow problems since the CPU of this hardware can only execute sequential tasks. However, some parallel platform like OpenMPI [10] are available for parallel computation in CPU, and the implementation paradigms are very complex. After the introduction of computing unified device architecture (CUDA) by Nvidia Corporation in 2007, the parallel computation in HPC became easier, and computation on parallel task execution units like GPU became possible for simulate fluid phenomena in a faster way than the convection CPU programming. In this paper, a GPU based parallel implementation of MRT based 3D coupled LBGK method is demonstrated for simulating the natural convection for a room having a partition inside the rectangular cavity.

\section{FORMULATION OF THE PROBLEM}

\section{Physical representation of the problem domain}

The natural convection in a rectangular enclosure with a heated wall boundary and a partition in a middle of the cavity is simulated using the MRT based LBM. The MRT-LBM code is validated under geometry of a cubic cavity, and the results are compared with the benchmark solutions. The schematic model is shown in Figure 1(a) with the dimension of the room was $0.914 m \times 0.914 m \times 0.914 m$ and a partition having $0.01 \mathrm{~m}$ width and $0.457 \mathrm{~m}$ height. The grid size of $180 \times 90 \times 90$ is been considered to simulation cases for two different Rayleigh number $(R a)$ of $R a=10^{4}$ and $R a=10^{5}$. The reference length of the physical geometry for the normalization procedure was the height of the room $H$ which has been used in the result and discussion section of this current demonstration.

\section{Governing Equations}

The evolution equation of LBM considers the fluid motion at macroscopic scale as distributing particle towards different discrete directions. The method uses discrete lattice model D3Q19 in Figure 1(b) which has 19 discrete position 


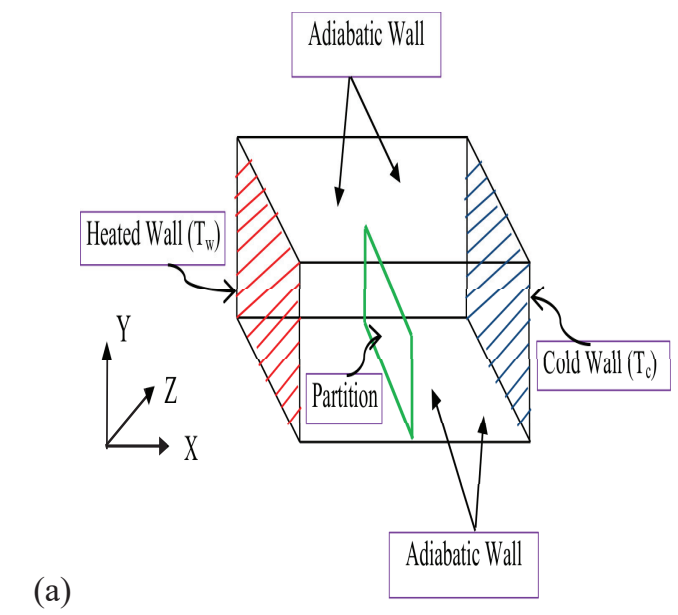

(b)

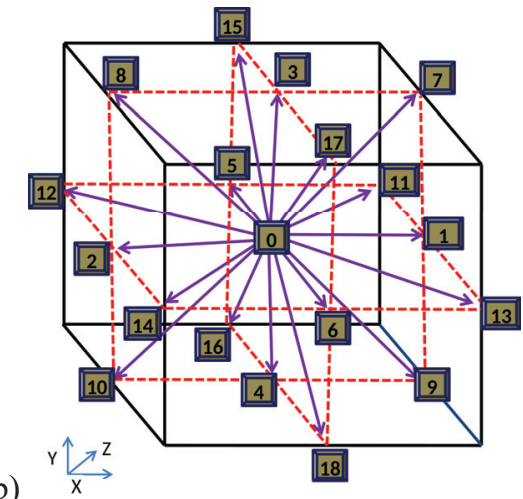

FIGURE 1. (a)Physical geometry of the problem (left) and (b) the D3Q19 lattice model of the MRT-LBM model (right)

vector for designating the directions of the fluid particles due to collision event. In LBM, the collision and streaming are the two major events for simulating the fluid flows. In collision process, fluid collides with each other and reaches to a equilibrium at a single or multiple characteristic times while they depend on the single or multiple relaxation parameter. The SRT based LBM methods considers the collision between fluid particles in the velocity space of the fluid regime while the MRT based approaches considers the process in the momentum space of the fluid continuity. The eqn. 1 is the formal evolution equation of the MRT based LBM method where distribution functions $\left(f_{\alpha} \mid \alpha=0,1,2,3, \ldots \ldots . ., Q-1\right)$ of $Q=19$ discrete velocities is as follows $[8,11]$ as follows:

$$
\mathbf{f}\left(x_{i}+e_{i} . \delta_{t}, t_{n}+\delta_{t}\right)=\mathbf{f}\left(x_{i}, t_{n}\right)-M^{-1} . \hat{S}\left(\mathbf{m}-\mathbf{m}^{\mathbf{e q}}\right)
$$

$\mathbf{M}=\left[\begin{array}{rrrrrrrrrrrrrrrrrrr}1 & 1 & 1 & 1 & 1 & 1 & 1 & 1 & 1 & 1 & 1 & 1 & 1 & 1 & 1 & 1 & 1 & 1 & 1 \\ -30 & -11 & -11 & -11 & -11 & -11 & -11 & 8 & 8 & 8 & 8 & 8 & 8 & 8 & 8 & 8 & 8 & 8 & 8 \\ -12 & -4 & -4 & -4 & -4 & -4 & -4 & 1 & 1 & 1 & 1 & 1 & 1 & 1 & 1 & 1 & 1 & 1 & 1 \\ 0 & 1 & -1 & 0 & 0 & 0 & 0 & 1 & -1 & 1 & -1 & 1 & -1 & 1 & -1 & 0 & 0 & 0 & 0 \\ 0 & -4 & 4 & 0 & 0 & 0 & 0 & 1 & -1 & 1 & -1 & 1 & -1 & 1 & -1 & 0 & 0 & 0 & 0 \\ 0 & 0 & 0 & 1 & -1 & 0 & 0 & 1 & -1 & -1 & 1 & 0 & 0 & 0 & 0 & 1 & -1 & 1 & -1 \\ 0 & 0 & 0 & 4 & -4 & 0 & 0 & 1 & -1 & -1 & 1 & 0 & 0 & 0 & 0 & 1 & -1 & 1 & -1 \\ 0 & 0 & 0 & 0 & 0 & 1 & -1 & 0 & 0 & 0 & 0 & 1 & -1 & -1 & 1 & 1 & -1 & -1 & 1 \\ 0 & 0 & 0 & 0 & 0 & 4 & -4 & 0 & 0 & 0 & 0 & 1 & -1 & -1 & 1 & 1 & -1 & -1 & 1 \\ 0 & 2 & 2 & -1 & -1 & -1 & -1 & 1 & 1 & 1 & 1 & 1 & 1 & 1 & 1 & -2 & -2 & -2 & -2 \\ 0 & 4 & 4 & 2 & 2 & 2 & 2 & 1 & 1 & 1 & 1 & 1 & 1 & 1 & 1 & -2 & -2 & -2 & -2 \\ 0 & 0 & 0 & 1 & 1 & -1 & -1 & 1 & 1 & 1 & 1 & -1 & -1 & -1 & -1 & 0 & 0 & 0 & 0 \\ 0 & 0 & 0 & -2 & -2 & 2 & 2 & 1 & 1 & 1 & 1 & -1 & -1 & -1 & -1 & 0 & 0 & 0 & 0 \\ 0 & 0 & 0 & 0 & 0 & 0 & 1 & 1 & -1 & -1 & 0 & 0 & 0 & 0 & 0 & 0 & 0 & 0 & 0 \\ 0 & 0 & 0 & 0 & 0 & 0 & 0 & 0 & 0 & 0 & 0 & 0 & 0 & 0 & 0 & 1 & 1 & -1 & -1 \\ 0 & 0 & 0 & 0 & 0 & 0 & 0 & 0 & 0 & 0 & 0 & 1 & 1 & -1 & -1 & 0 & 0 & 0 & 0 \\ 0 & 0 & 0 & 0 & 0 & 0 & 0 & 1 & -1 & 1 & -1 & -1 & 1 & -1 & -1 & 0 & 0 & 0 & 0 \\ 0 & 0 & 0 & 0 & 0 & 0 & 0 & -1 & 1 & 1 & -1 & 0 & 0 & 0 & 0 & 1 & -1 & 1 & -1 \\ 0 & 0 & 0 & 0 & 0 & 0 & 0 & 0 & 0 & 0 & 0 & 1 & -1 & -1 & 1 & -1 & 1 & 1 & -1\end{array}\right]$

Here, the $\mathbf{f}$ is the linear transformation of the $Q$ matrix $\mathbf{M}$ while $\mathbf{m}$ and $\mathbf{m}{ }^{\mathbf{e q}}$ are the moments in moments space $M \in R^{Q}$. The collision between particles in MRT-LBM is performed in the moment space where collision matrix $\hat{S}$ for different $m$ moments for each discrete velocities of velocity model. Then advection is performed in the velocity space $V \in R^{Q}$ through the $\mathbf{f}$ moments. The advection and collision terms of Eqn. 1 can be computed as follows:

$$
\begin{gathered}
\mathbf{f}\left(x_{i}+e_{i} . \delta t, t_{n}+\delta_{t}\right):=\left(f_{0}\left(x_{i}+e_{i} . \delta_{t}, t_{n}+\delta_{t}\right), \ldots \ldots, f_{Q}\left(x_{i}+e_{Q} \delta_{t}, t_{n}+\delta_{t}\right)\right)^{T} \\
\mathbf{f}\left(x_{i}, t_{n}\right):=\left(f_{0}\left(x_{i}, t_{n}+\delta_{t}\right), \ldots \ldots ., f_{Q}\left(x_{i}, t_{n}+\delta_{t}\right)\right)^{T} \\
\mathbf{m}:=\left(m_{0}\left(x_{i}, t_{n}\right), m_{1}\left(x_{i}, t_{n}\right), \ldots \ldots \ldots, m_{Q}\left(x_{i}, t_{n}\right)\right)^{T} \\
\mathbf{m}^{(\mathbf{e q})}:=\left(m_{0}^{(e q)}\left(x_{i}, t_{n}\right), m_{1}{ }^{(e q)}\left(x_{i}, t_{n}\right), \ldots \ldots \ldots, m_{Q}(e q)\left(x_{i}, t_{n}\right)\right)^{T}
\end{gathered}
$$


TABLE 1. Comparison of the Average Nusselt number $\overline{N u}$ of present MRT-LBM code with benchmark results

\begin{tabular}{|l|l|l|l|l|}
\hline $\mathrm{Ra}$ & {$[12]$} & {$[13]$} & {$[14]$} & $96 \times 96 \times 96$ \\
\hline $10^{4}$ & 2.05 & 2.06 & 2.08 & 2.02 \\
\hline $10^{5}$ & 4.34 & 4.38 & 4.36 & 4.24 \\
\hline
\end{tabular}

Here, $T$ is transpose operator. In $D 3 Q 19$ lattice model the velocities of the evolution equation (Eqn. 1) are defined as follows:

$$
\begin{gathered}
\mathbf{e}_{\mathbf{i}}=(0,0,0) i=0 \\
( \pm 1,0,0),(0, \pm 1,0),(0,0, \pm 1) i=1-6 \\
( \pm 1, \pm 1,0),( \pm 1,0, \pm 1),(0, \pm 1, \pm 1) i=7-18
\end{gathered}
$$

The $\mathbf{m}^{(e q)}$ is the non-conserved moments equilibrium which depends on the density fluctuations $(\delta \rho)$, energy $(\epsilon)$, momentum $\left(\mathbf{j}=\mathbf{j}_{\mathbf{x}}, \mathbf{j}_{\mathbf{y}}, \mathbf{j}_{\mathbf{z}}\right)$ and heat flux $\left(\mathbf{q}=\mathbf{q}_{\mathbf{x}}, \mathbf{q}_{\mathbf{y}}, \mathbf{q}_{\mathbf{z}}\right)$ [11]. The 19 different moments of the D3Q19 lattice model for MRT-LBM are crucial for defining the relaxation matrix $\hat{S}$. The relaxation matrix $\hat{S}$ which is the diagonal matrix of the moment space $\mathbf{M}$ as well as the moments equilibrium functions can be written as follows:

$$
\begin{gathered}
\hat{S}=\operatorname{diag}\left(1,1.19,1.4,1,1.2,1,1.2,1,1.2, s_{9}, 1.4, s_{9}, 1.4, s_{9}, s_{9}, s_{9}, 1.98,1.98,1.98\right) \\
m_{1}{ }^{(e q)}=-11 \delta \rho+\frac{19}{\rho_{0}} \cdot j \cdot j \\
m_{2}{ }^{(e q)}=\omega_{\epsilon} \delta \rho+\frac{\omega_{\epsilon}}{\rho_{0}} \mathbf{j} \cdot \mathbf{j} \\
m_{4,6,8}{ }^{(e q)}=\frac{-2}{3} j_{x, y, z} \\
m_{9}^{(e q)}=\frac{1}{\rho_{0}}\left(3 j^{2}{ }_{x}-\mathbf{j} \cdot \mathbf{j}\right) \\
m_{11}{ }^{(e q)}=\frac{1}{\rho_{0}}\left(j^{2}{ }_{y}-j_{z}^{2}\right) \\
m_{10}{ }^{(e q)}=\omega_{x x}, m_{9}{ }^{(e q)} \\
m_{12}{ }^{(e q)}=\omega_{x x} m_{11}{ }^{(e q)} \\
m_{13}{ }^{(e q)}=\frac{1}{\rho_{0}} j_{x} \cdot j_{y} \\
m_{14}{ }^{(e q)}=\frac{1}{\rho_{0}} j_{y} \cdot j_{z} \\
m_{15}{ }^{(e q)}=\frac{1}{\rho_{0}} j_{z} \cdot j_{x} \\
m_{16}{ }^{(e q)}=m_{17}{ }^{(e q)}=m_{18}{ }^{(e q)}=0
\end{gathered}
$$

The viscosity of the fluid is related with the

$$
v=\frac{1}{3}\left(\frac{1}{s_{9}}-\frac{1}{2}\right) c^{2} \Delta t \quad c=\frac{\Delta x}{\Delta t}=1
$$


TABLE 2. Grid independence test in terms of the Average Nusselt number $\overline{N u}$ for two different grid arrangements.

\begin{tabular}{|l|l|l|}
\hline $\mathrm{Ra}$ & $120 \times 60 \times 60$ & $180 \times 90 \times 90$ \\
\hline $10^{4}$ & 2.6143 & 2.7322 \\
\hline $10^{5}$ & 5.9492 & 5.9819 \\
\hline
\end{tabular}
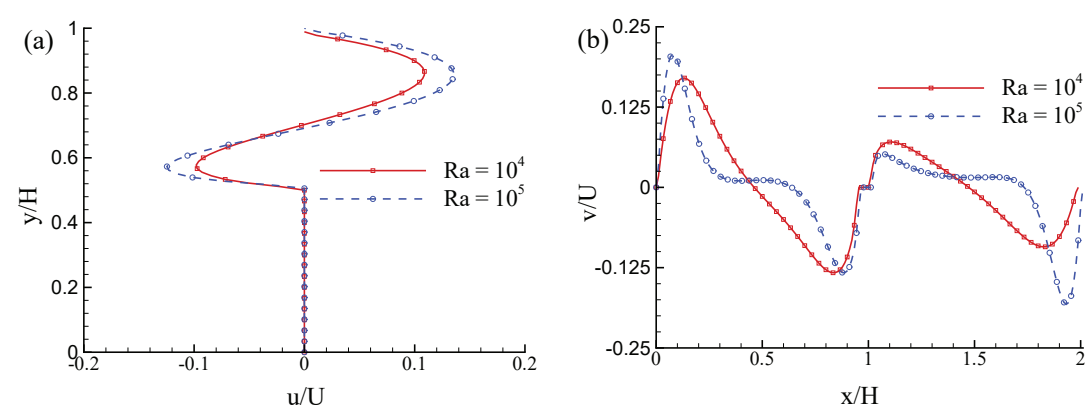

FIGURE 2. Velocity distribution (a) $u$ velocity at $x / H=0.5$ and (b) $v$ velocity at $y / H=0.5$ while $z / H=0.5$.

\section{RESULTS AND DISCUSSION}

To validate the GPU based MRT-LBM code, the natural convection for a 3D cubic cavity has been studied in terms of average Nusselt number $(\overline{\mathrm{Nu}})$ that is shown in Table 1 comparing with the results of [12-14] and found excellent agreement. For the code validation, the lattice size of $(96 \times 96 \times 96)$ is chosen to simulate the natural convection. After the code validation the natural convection in a rectangular cavity with a partition is shown in Figure 1(b) is simulated for $R a=10^{4}$ and $R a=10^{5}$. The partition is located at the middle of the cavity, and the height is half $(0.5 \mathrm{H})$ of the cavity height. The results are presented in terms of the velocity and temperature distribution, average Nusselt number as well as streamlines and isotherms for two different Rayleigh numbers. Firstly, Table 2 shows the grid sensitivity test in terms of the average Nusselt number $\overline{N u}$ with two lattice sizes $120 \times 60 \times 60$ and $180 \times 90 \times 90$. The results indicate that two sets of the grid are sufficient to simulate the present problem. Here $180 \times 90 \times 90$ lattice size is chosen for the rest of the computation. Here it can also be summarised that the average rate of heat transfer increases while $R a$ increases.

The horizontal and vertical velocity distribution for the different different Rayleigh numbers $\left(R a=10^{4}, 10^{5}\right)$ are depicted in Figures 2(a)-(b) respectively at the mid of span-wise plane, $z / H=0.5$. From these figures, it is clearly seen that the velocity increase owing to the increase of $R a$. In Figures 2(a), the $u / U$ velocity is zero from $y / H=0$ to 0.5 due to the presence of partition and in frame (b), it is ensured that the maximum and minimum $v / U$ velocity occurred neat the hot and cold wall. Here the normalized velocity $U$ is $\sqrt{g \beta \Delta H}$. Figure 3(a)-(b) show the corresponding temperature distribution at $x / H=0.5$ and $y / H=0.5$, respectively. For the variation of $R a$, the temperature distribution vary significantly.

Figure 4(a)-(b) represents the isotherms at mid $x y$-plane of the cavity with partition where gradual temperature distribution is observed for both $R a=10^{4}$ and $R a=10^{5}$, respectively. In the figure, it is clear that the temperature is gradually distributed from the hot wall to the cold wall and the temperature has a higher magnitude near the left wall and propagated to the cold right wall. It can also be concluded that the temperature distribution in natural convection can be hindered due to the presence of a partition. The corresponding streamlines appended in $u / U$ velocity are illustrated in Figures 5(a)-(b). Due to the presence of the partition, the flow pattern changes and formed two large vortex in the two side of the partitions. The maximum and minimum velocity $u / U$ is 0.16 and -0.13 while $R a=10^{4}$ but the maximum and minimum velocity is 0.15 and -0.14 at the case of $R a=10^{5}$.

Figure 6 represents the temperature iso-surfaces of $3 D$ of the cavity having one partition at the middle of the room at $\mathrm{Ra}=10^{4}$. In the figure, the convection is hindered due to the presence of the partition and the cold areas remains cold near the cold wall. On the other hand,hot airflow of the convective flow distributed naturally at the top of the partition. 

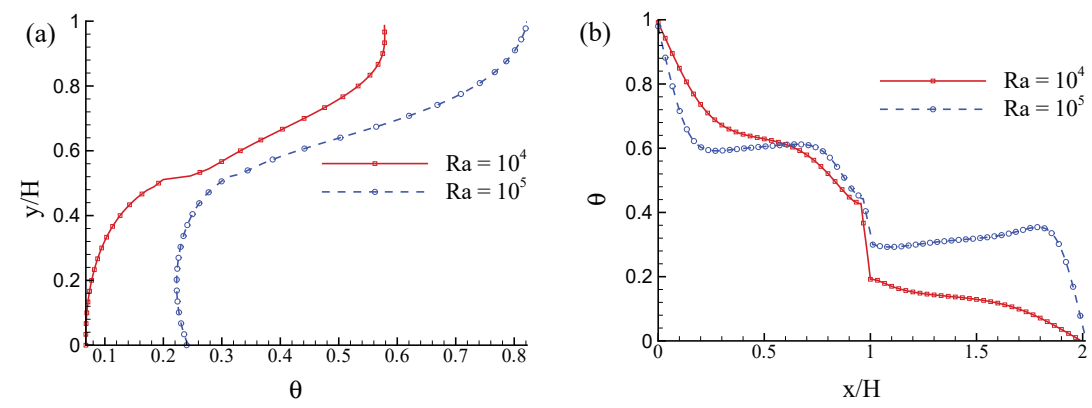

FIGURE 3. Temperature distribution at (a) $x / H 0.5$ and (b) $y / H=0.5$ while $z / H=0.5$.
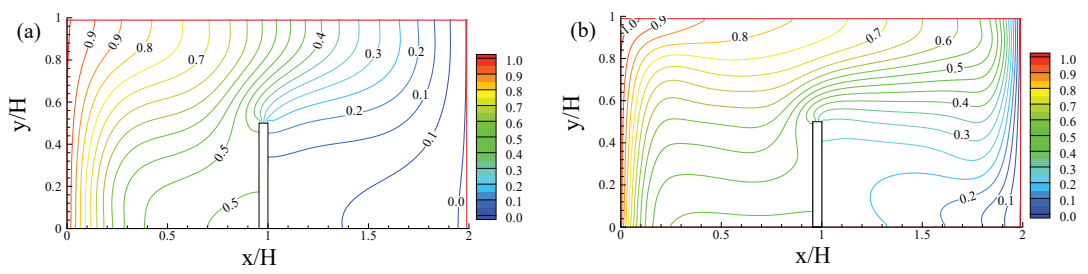

FIGURE 4. Isotherms for (a) $R a=10^{4}$ and (b) $R a=10^{5}$ at $z / H=0.5$.

\section{CONCLUSION}

In this paper, we presented a GPU based MRT-LBM implementation for the simulation of the natural convection of a rectangular cavity using $D 3 Q 19$ lattice model for velocity and $D 3 Q 6$ coupled lattice model for temperature. We found a considerable amount of efficiency over existing GPU based LBM simulations and also the implementations using CPU. The GPU based parallel CUDA C code is validated for the $3 D$ cubic cavity with the benchmark solutions. The results are at great accord with the existing literature. Then the convective flow in a rectangular $3 D$ cavity with a partition is simulated for two different $R a$, and we found the effect of the partition in convective heat transfer. For $R a=10^{5}$, the average rate of heat transfer with and without partition are 5.9819 and 6.9471 , respectively.

The convective flow in a real-world room using the LBM based solutions can interpret the thermal comforts in an urban building and more solutions on civil engineering problems. Several researchers $[16,17]$ implemented LBM in GPU based hardware and Obrecht et al. [16] found significant results of using maximum $86 \%$ of the global DRAM (Dynamic random access memory) using the GPU based LBM code which is hardly found on the traditional sequential CPU computing. Delbosc et al. [17] implemented memory efficient GPU code and found 6\% efficiency compared to the unorganized PULL-OUT scheme of LBM. We implemented PULL-IN scheme of GPU based LBM code for efficient memory usage and achieved $7.32 \%$ efficiency over the PULL-OUT scheme.

In the present LBM code, we have not used shared memory and we do believe that we can accelerate more by using shared memory technique in this GPU based MRT-LBM code. In our future research, adopting shared memory approach we will investigate the turbulent air flow and heat transfer by using the large-eddy simulation (LES) technique in this MRT based lattice Boltzmann method. 

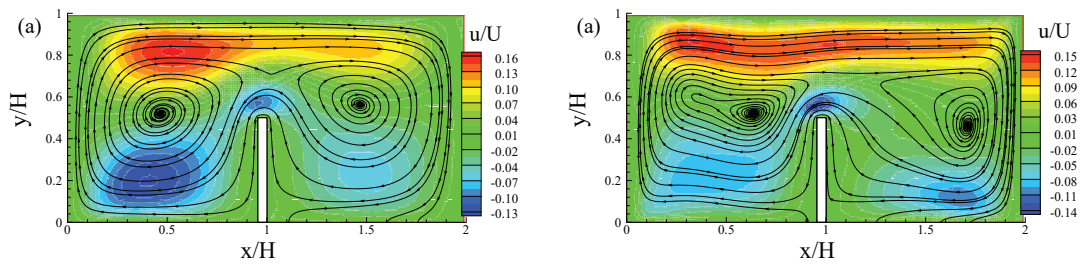

FIGURE 5. Streamlines appended on $u / U$ velocity for (a) $R a=10^{4}$ and (b) $R a=10^{5}$ at $z / H=0.5$.

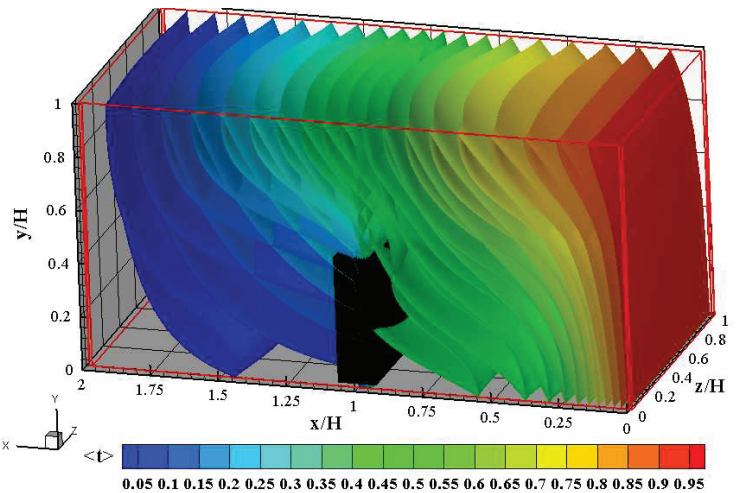

FIGURE 6. $3 \mathrm{D}$ view of temperature iso-surfaces of the rectangular cavity with a partition while $\mathrm{Ra}=10^{4}$.

\section{REFERENCES}

[1] Y. Tian and T. Karayiannis, International Journal of Heat and Mass Transfer 43, 849-866 (2000).

[2] W. Leong, K. Hollands, and A. Brunger, International Journal of Heat and Mass Transfer 42, 1979-1989 (1999).

[3] U. Frisch, B. Hasslacher, and Y. Pomeau, Physical review letters 56, p. 1505 (1986).

[4] D. A. Wolf-Gladrow, Lattice-gas cellular automata and lattice Boltzmann models: an introduction (Springer, 2004).

[5] Z. Guo, B. Shi, and N. Wang, Journal of Computational Physics 165, 288-306 (2000).

[6] Z. Guo, B. Shi, and C. Zheng, International Journal for Numerical Methods in Fluids 39, 325-342 (2002).

[7] P. L. Bhatnagar, E. P. Gross, and M. Krook, Physical review 94, p. 511 (1954).

[8] D. d'Humières, Philosophical Transactions of the Royal Society of London A: Mathematical, Physical and Engineering Sciences 360, 437-451 (2002).

[9] M. Molla, M. Haque, M. Khan, and S. Saha, Front. Mech. Eng. 4, 1-15 (2018).

[10] E. Gabriel, G. E. Fagg, G. Bosilca, T. Angskun, J. J. Dongarra, J. M. Squyres, V. Sahay, P. Kambadur, B. Barrett, A. Lumsdaine, et al., "Open mpi: Goals, concept, and design of a next generation mpi implementation," in European Parallel Virtual Machine/Message Passing Interface Users Group Meeting (Springer, 2004), pp. 97-104.

[11] H. Yu, L.-S. Luo, and S. S. Girimaji, Computers \& Fluids 35, 957-965 (2006).

[12] E. Tric, G. Labrosse, and M. Betrouni, International Journal of Heat and Mass Transfer 43, 4043-4056 (2000).

[13] S. Wakashima and T. S. Saitoh, International Journal of Heat and Mass Transfer 47, 853-864 (2004).

[14] Y. Peng, C. Shu, and Y. Chew, Journal of Computational Physics 193, 260-274 (2004).

[15] T. Fusegi, J. M. Hyun, K. Kuwahara, and B. Farouk, International Journal of Heat and Mass Transfer 34, 1543-1557 (1991).

[16] C. Obrecht, F. Kuznik, B. Tourancheau, and J.-J. Roux, Computers \& Mathematics with Applications 61, 3628-3638 (2011).

[17] N. Delbosc, J. L. Summers, A. Khan, N. Kapur, and C. J. Noakes, Computers \& Mathematics with Applications 67, 462-475 (2014). 